\title{
Comparison of Reactive Flow Simulations for a LOX/CH4 Multi-element Rocket Engine
}

\author{
Ainslie D. French*, Luigi Cutrone, Antonio Schettino, Marco M arini, Francesco Battista \\ and Pasquale $\mathrm{N}^{2}$ atale $\mathrm{e}^{1}$ \\ CIRA (Italian A erospace Research Cemter), V ia M aiorise, 81943 Capua (CE), Italy
}

\begin{abstract}
This study details the reactive flow simulations of a $\mathrm{LOX} / \mathrm{CH} 4$ $\mathrm{M}$ ulti-element rocket engine. The work has been conducted within the framework of the HYPROB-BREAD project whose main objective is the design, manufacture and testing of a $\mathrm{LOX} / \mathrm{LCH} 4$ regeneratively cooled ground demonstrator. Numerical simulations have been carried out with both commercial software and CIRA software developed in house. Two sets of boundary conditions, nominal and experimental, have been applied from which a code-to-code validation has been effected with the former and a code-to-experiment validation with the latter. The results presented include both flow data and heat fluxes as well as parameters associated with engine performance, and indicate an excellent agreement with experimental data of a LOX/CH4 M ulti-element rocket engine.
\end{abstract}

\section{Introduction}

Turbulent combustion is an extremely complicated physical process to model. Analytical techniques are inadequate for modelling such complex processes. A plethora of commercial codes have been developed which are potentially suitable to model such problems. In this paper ANSYS-FLUENT ${ }^{\circledR}$ version 14.5 [1] and NExT are used to model the turbulent compressible reacting flow field within the HYPROB-BREAD LOX/CH4 multi-element rocket, the details of which are described in [2].

This rocket engine utilizes a regeneratively cooled thrust chamber for ground testing at $30 \mathrm{KN}$ thrust. A counter-flow architecture is used for the chamber nozzle (2) cooling system, as shown in Figure 1, where the methane, serving as coolant, is injected into the fuel inlet (1) and collected in the manifold which then distributes the methane through cooling channels in the cooling jacket in the counter-flow direction relative to the combustion gases, see Figure 2.

\footnotetext{
* Corresponding author: a.french@cira.it
} 


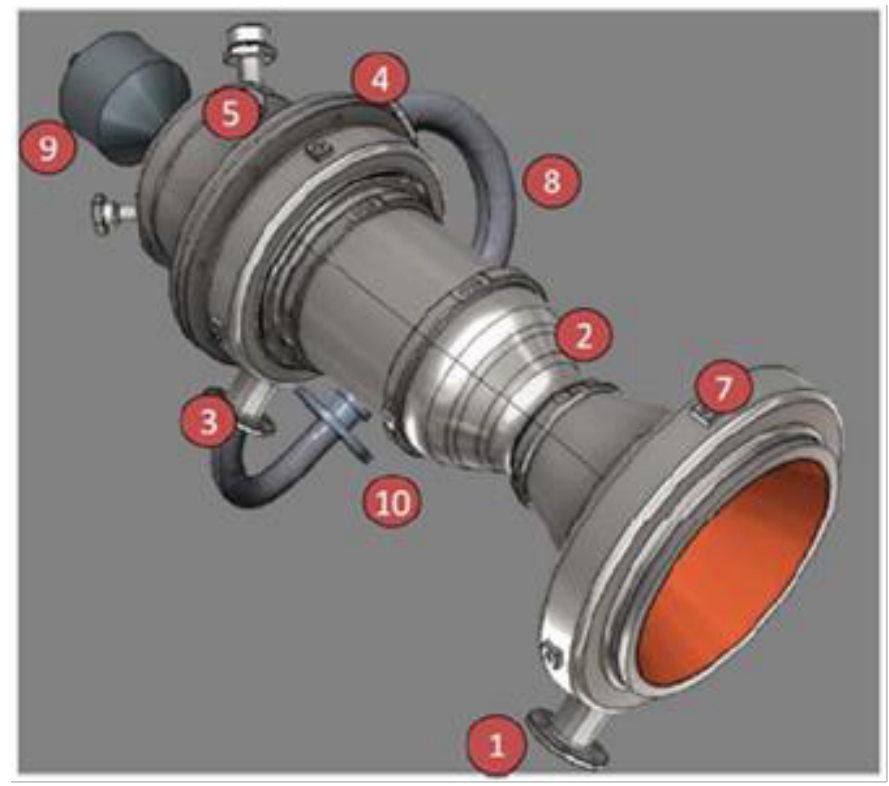

Fig. 1. HYPROB-BREAD LOX/CH4 Rocket Engine.

After being heated by the combustion gases the methane passes through a valve and tubing system (3-10) before being injected directly into the fuel dome (4). From there the coolant is injected into the chamber where it atomizes, mixes with the oxygen and burns.

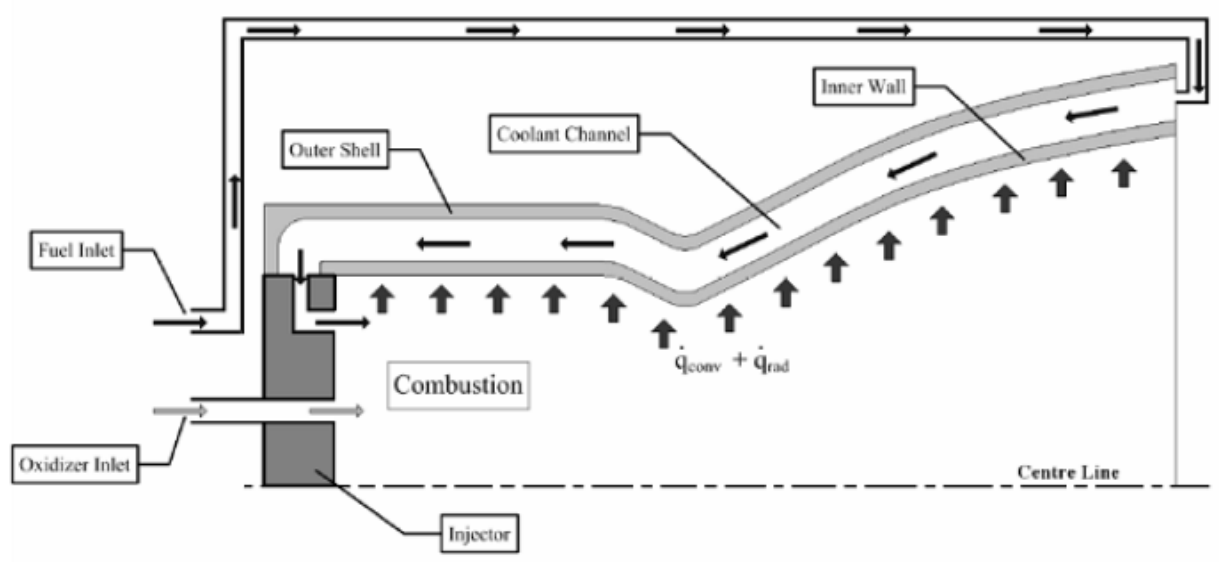

Fig. 2. Regeneratively cooled combustion chamber.

Two sets of inflow boundary conditions were applied corresponding to the design demonstrator (DEMO) nominal conditions associated with regenerative cooling, and the experimental conditions of a full-scale breadboard (FSBB) using a water cooled combustion chamber tested in the AVIO FAST2 test facility at Colleferro, Rome, in summer 2017. 


\section{Numerical Simulation Methodology}

\subsection{Domain and Grid Parameters}

All calculations were performed on the same fine grid representing one sixth of the original configuration where the cluster of three injectors is symmetrical. The grid consisted of about 3.5 million cells and was interpreted as an unstructured grid for the ANSY SFLUENT calculation, and was converted into a fine and coarse 3D multiblock grids having 766 blocks and 3.5 and 1.75 million cells, respectively, for the NExT calculation, see Figure 3. The grids were generated with the ANSY S ${ }^{v}$ ICEM CFD ${ }^{\mathrm{TM}}$ software and satisfy the associated quality criteria.
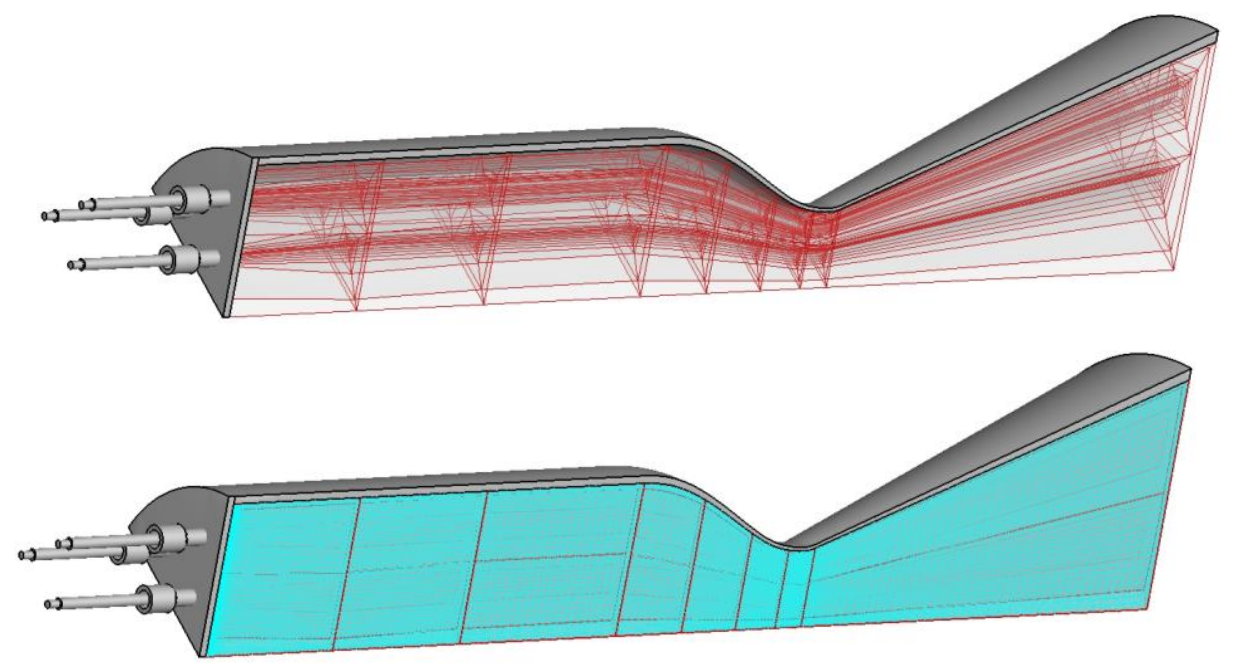

Fig. 3. Grid showing multiblock structure used in NEXT.

\subsection{Solver and Boundary Conditions}

For the ANSY S-FLUENT ${ }^{v}$ calculations the compressible 3D Navier-Stokes equations were solved with the pressure based solver using a coupled scheme. Pseudo transient timestepping was employed combined with the standard $k-\epsilon$ turbulence model with the standard wall function to model turbulence. The reaction mechanisms were modelled for non-premixed combustion using species transport and the Eddy Dissipation M odel (EDM) for the chemical kinetics.

A single-step kinetic scheme mechanism which includes the species $\mathrm{CH} 4, \mathrm{O} 2, \mathrm{H} 2 \mathrm{O}$, and C02 was adopted.

The specific heat at constant pressure was calculated using the mixing law, the thermal conductivity and viscosity are calculated using the ideal gas mixing law and the mass diffusivity using kinetic theory. The critical temperature, pressure, specific volume were calculated using the one fluid $V$ an der $W$ aals mixing law and the acentric factor used the mole-weighted mixing law. The chemical reactions were relaxed to equilibrium conditions.

The calculations were performed to second order accuracy with the criteria for convergence being negligible difference in mass flux between inflow and outflow.

In the ANSY S-FLUENT ${ }^{v}$ calculation the standard state methane enthalpy was adjusted from its true value of $-7.49 .10^{7} \mathrm{~J} / \mathrm{kgmol}$ to $-3.5 .10^{8} \mathrm{~J} / \mathrm{kgmol}$ in order to obtain more 
physically realistic adiabatic flame temperatures which would otherwise be excessively high.

The NEXT CIRA code also has solved the 3D compressible Navier-Stokes equations using a time marching point implicit scheme for the integration to approach the steady state. The convective scheme used second order upwind flux differencing and turbulence was modelled with the standard $\mathrm{k}-\epsilon$ turbulence model with the standard wall function.

For the chemical kinetics a single-step kinetic scheme equivalent to the one used in the ANSYS-FLUENT $^{v}$ calculation was implemented. The reaction mechanisms were modelled for non-premixed combustion using species transport and the eddy dissipation model for the chemical kinetics.

The calculations were divided into two sets, the first set corresponding to a direct comparison of results between ANSY S-FLUENT ${ }^{\circledR}$ and NExT for "nominal" or "DEMO" test conditions associated with the exact theoretical values which should be applied to the demonstrator, and then a second set of "experimental" or "FSBB" conditions corresponding to the actual values used in the A VIO FAST2 facility experiment.

ANSY S-FLUENT ${ }^{\vee}$ used the Soave Redlich K wong equation for the density calculation whereas NEXT used the ideal gas equation of state.

The inner injector jet carried oxygen whereas the outer annulus carried methane. For the nominal test case a mixture ratio of 3.4 was used together with an oxidizer mass flow rate of $1.089 \mathrm{~kg} / \mathrm{s}$ at $110 \mathrm{~K}$ and a fuel temperature of $450 \mathrm{~K}$ at pressures of $6 \mathrm{M} \mathrm{Pa}$. In the experimental test case a mixture ratio of 3.21 was used together with an oxidizer mass flow rate of $0.982 \mathrm{~kg} / \mathrm{s}$ at $129 \mathrm{~K}$ and a fuel temperature of $292 \mathrm{~K}$ and pressures of $6.1 \mathrm{M} \mathrm{Pa}$ and 7.5 $\mathrm{MPa}$, respectively. The chamber and nozzle wall temperatures were set to $450 \mathrm{~K}$ in both cases.

\subsection{Numerical results}

\subsubsection{Nominal DEMO test case}

A comparison of the axial pressure distributions between NExT and ANSYSFLUENT ${ }^{v}$ for solutions on a fine grid is shown in Figure 4 below.

The results are generally in good agreement, where the ANSYS-FLUENT ${ }^{v}$ chamber pressure predictions are only about $5 \%$ higher than those of NEXT. B oth simulated pressure distributions are below and outside the error-bar of the experimental value cited in [3].

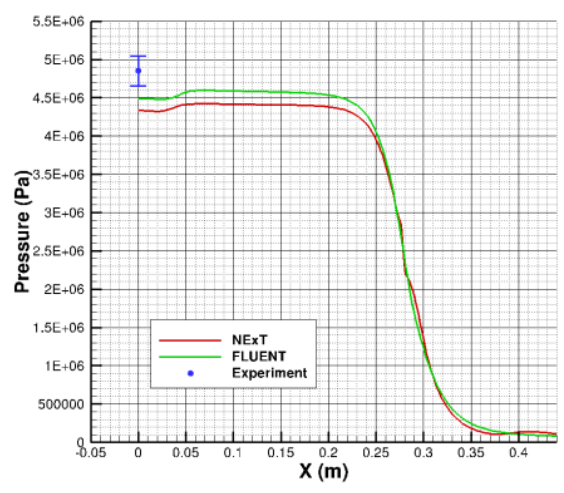

Fig. 4. Comparison of axial pressure distributions on fine grid. 
A comparison of pressure contours between NEXT and ANSY S-FLUENT ${ }^{v}$ taken on a slice through two injectors is shown in Figure 5 below.

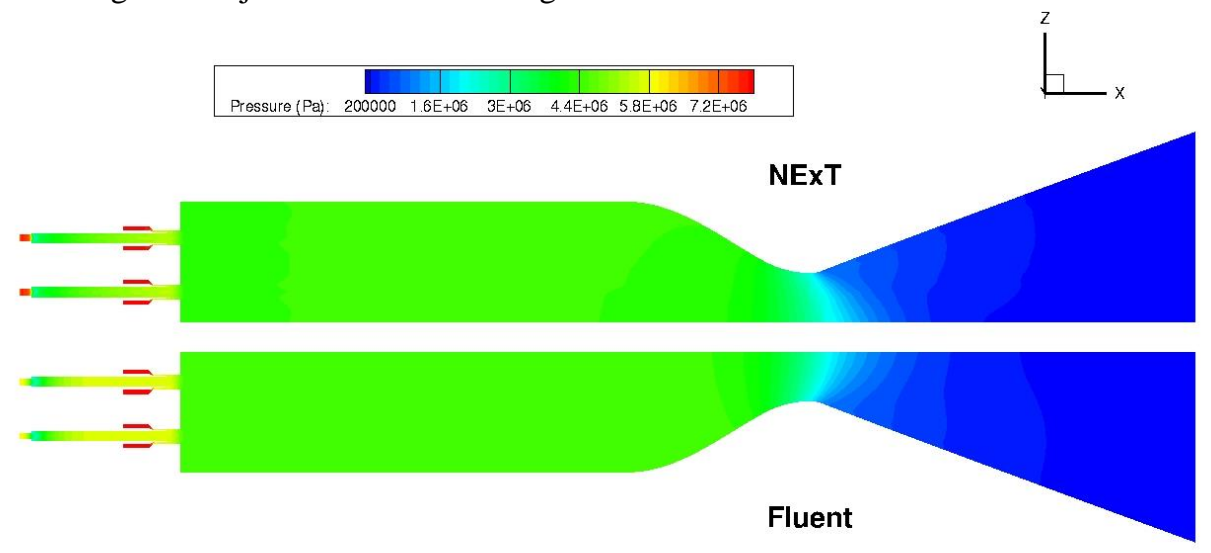

Fig. 5. Comparison of pressure contours on slice for NEXT and FLUENT on fine grid.

A comparison of temperature contours between NExT and ANSY S-FLUENT ${ }^{v}$ taken on a slice through two injectors is shown in Figure 6 below. The temperature contours are in reasonable agreement, the NEXT simulation having a longer and cooler stream of LOX and a larger and hotter region of combusting gases near the wall than in the ANSYSFLUENT $^{\vee}$ simulation.

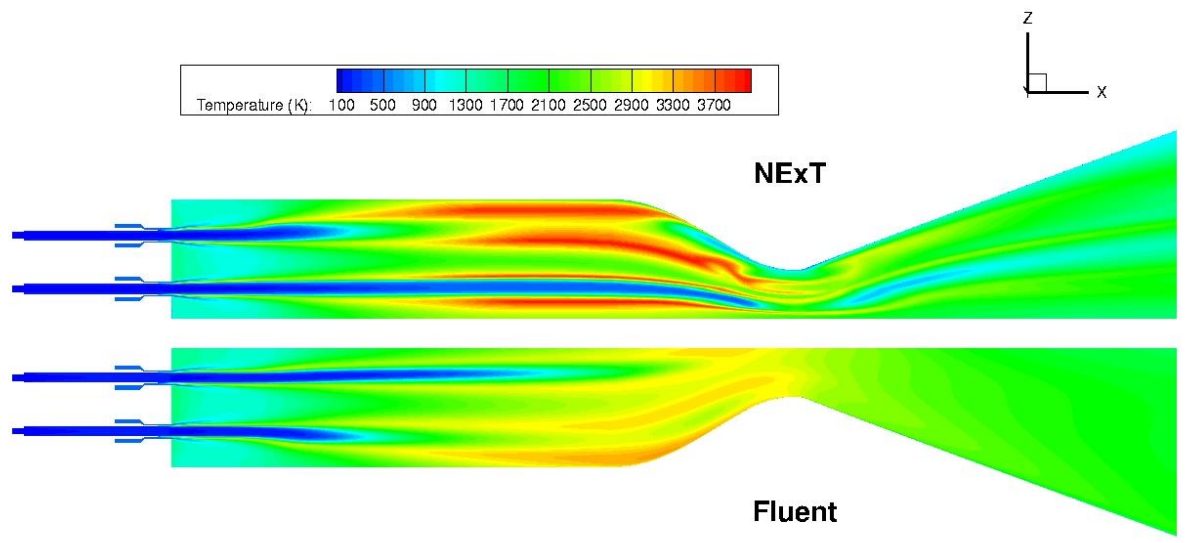

Fig. 6. Comparison of temperature contours on slice for NEXT and FLUENT on fine grid.

\subsubsection{Experimental FSBB test case}

A comparison of NEXT solutions for axial pressure distributions on fine and coarse grids is shown in Figure 7 below. There is excellent agreement between the chamber pressure predictions on both grids as well as with the experimental value measured by the igniter chamber pressure sensor. 


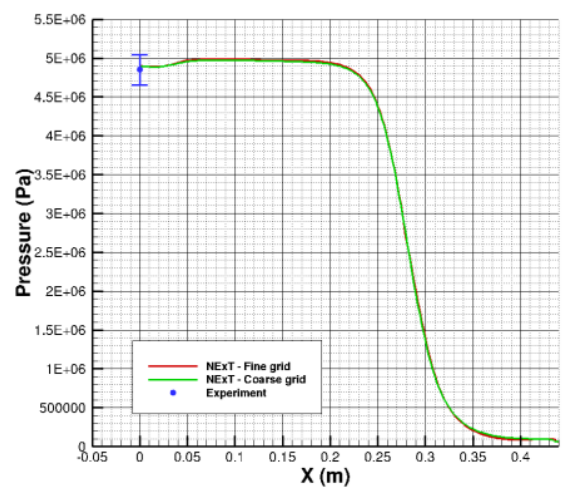

Fig. 7. Comparison of axial pressure distributions for NExT on fine and coarse grids.

Subsequent results show a comparison of pressure and temperature contours represented as isolines on the same slice taken through two injectors as presented for the nominal test case. In all cases, there is excellent agreement in the results on both grids.

A comparison of pressure contours for NEXT on fine and coarse grids taken on a slice through two injectors is shown in Figure 8 below.

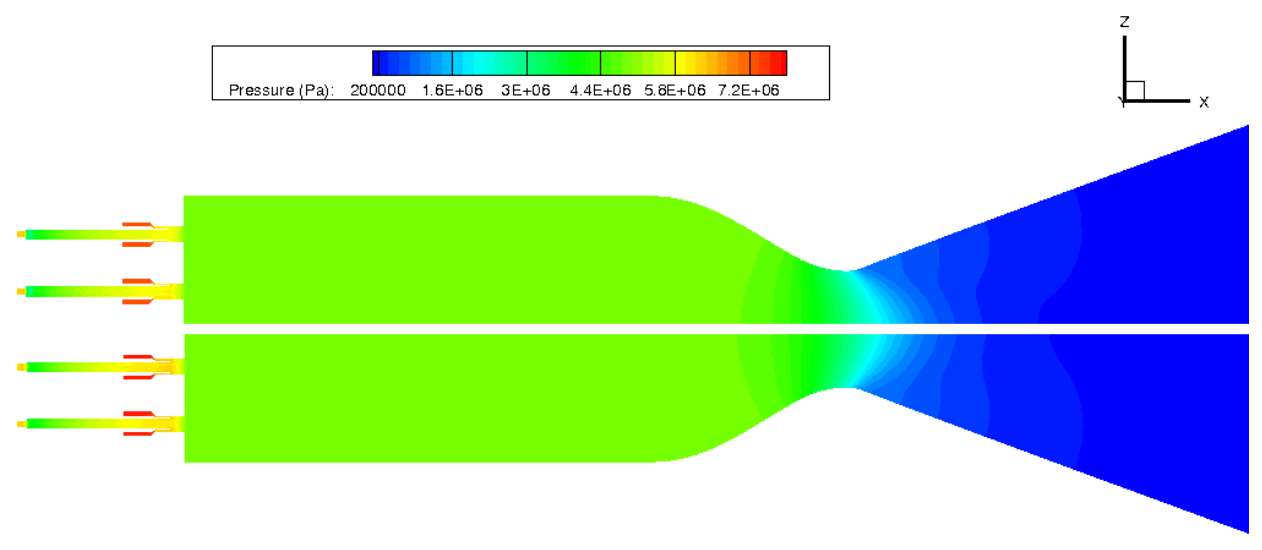

Fig. 8. Comparison of pressure contours for NEXT on fine (upper) and coarse (lower) grids.

A comparison of temperature contours for NEXT on fine and coarse grids taken on a slice through two injectors is shown in Figure 9 below. 


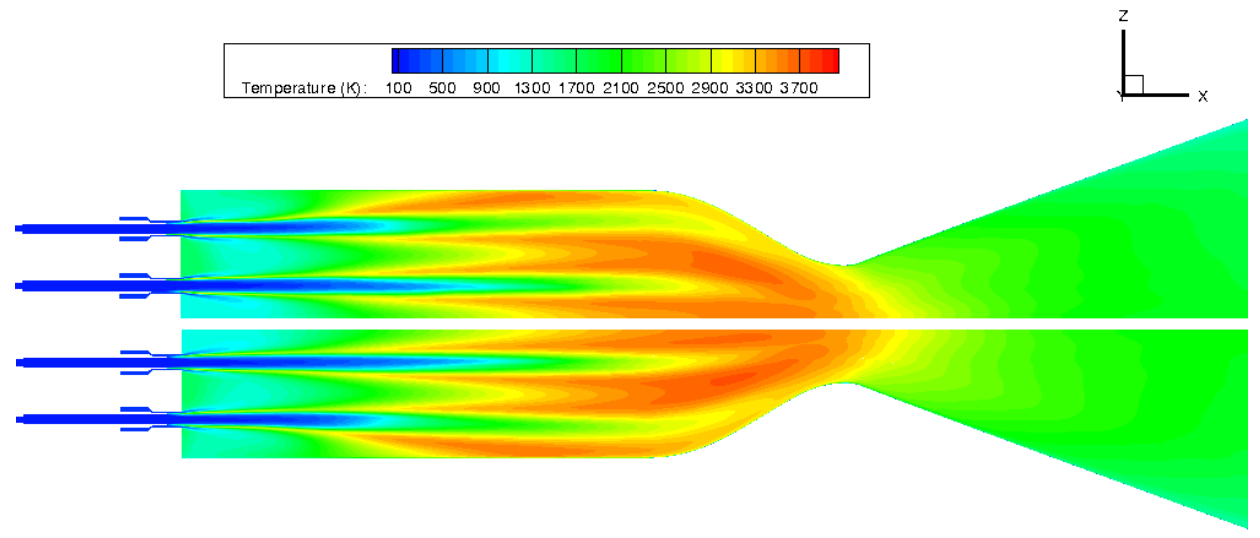

Fig. 9. Comparison of temperature contours for NEXT on fine (upper) and coarse (lower) grids.

A comparison of heat fluxes predicted by ANSYS-FLUENT ${ }^{\vee}$ for the nominal DEMO test case and by NEXT for the experimental FSBB test case at 15-degree slice locations is shown in Figure 10.
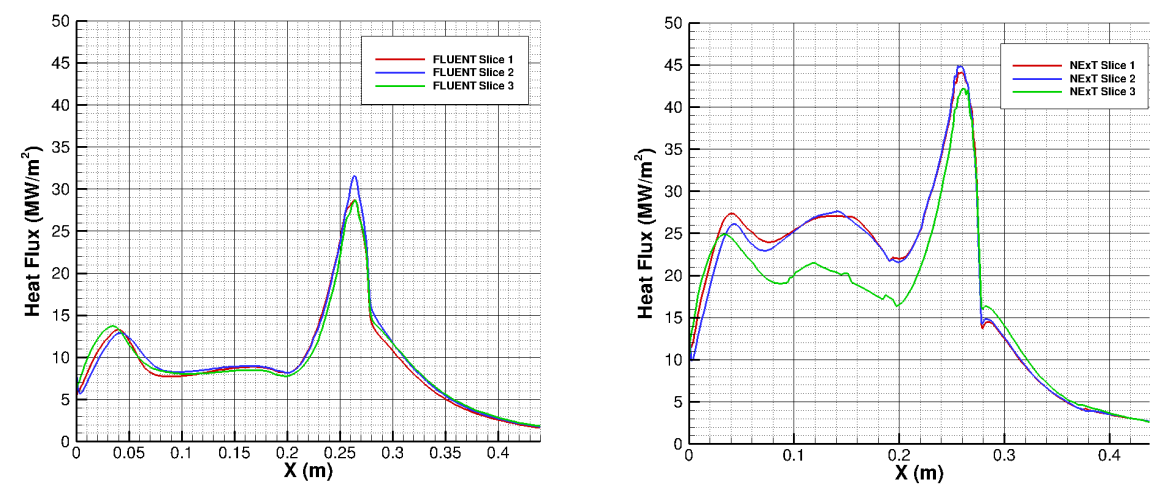

Fig. 10. Heat flux distributions for nominal ANSYS-FLUENT ${ }^{v}$ (left) and experimental NExT (right) test cases.

The peak heat fluxes at the nozzle throat are about 50\% higher for the experimental FSB B test case than for the nominal DEM 0 test case.

A comparison of thrust predictions for the DEM 0 indicated values of $19.13 \mathrm{KN}$ with NEXT compared with $19.78 \mathrm{KN}$, corresponding to the experimental value, with FLUENT. For the FSB B a thrust of $20.62 \mathrm{KN}$ was predicted by NEXT, which was $4 \%$ higher than the experimental value, compared to $17.70 \mathrm{KN}$ predicted by FLUENT which was $11 \%$ lower than the experimental value.

Regarding combustion efficiency for the DEMO, NEXT predicted $80 \%$ efficiency compared to $83 \%$ with FLUENT, whereas for the FSBB condition NExT predicted $97 \%$ efficiency being in excellent agreement with the experimental value of $96 \%$ compared to $84 \%$ predicted by FLUENT.

NEXT also demonstrated excellent performance where the fine grid calculation took 30 days and the coarse grid calculation 3 days, both on 36 CPU s compared to estimated values of 50 and 7 days, respectively. 


\section{Concluding Remarks}

The nominal test case indicated good agreement in chamber pressures between the commercial software, ANSY S-FLUENT ${ }^{v}$ and the CIRA in house software NEXT, where both codes predicted lower chamber pressures than in the experimental test case due to slightly different operating conditions.

NEXT exceeded performance requirements for both fine and coarse grids whereas the quality of the results was preserved.

A superior level of combustion is evident in the experimental test case compared to the nominal test case. The explanation for this difference is most likely associated with the effect of the inlet conditions. In particular, with respect to the nominal conditions, the main differences in the experimental conditions are a slightly smaller mixture ratio and a lower methane inlet temperature.

Comparison of thrust and combustion efficiency indicates that NEXT is superior to FLUENT having thrust and combustion efficiencies of $4 \%$ and $1 \%$ higher than experiment compared to $7 \%$ and $2 \%$ with FLUENT, respectively.

Future work in NEXT will include a systematic investigation of the differences in combustion efficiency by gradually changing the inlet conditions as well as an improvement of the mixing model in order to have more efficient combustion of oxygen.

The HYPROB program is a major element of the CIRA A erospace Propulsion Program to support the development of Space Propulsion in coherence with the Italian National long-term vision defined by the Italian Space A gency (A SI).

The program is carried out under contract by the Italian M inistry of University and Research (MIUR).

\section{References}

1. ANSY S FLUENT User's Guide, release 14.5, ANSY S Inc., Canonsburg, Pennsylvania, USA.

2. Cardillo, D., Panelli, P., Ricci, D., Roncioni, P., B attista, F. "Numerical Simulation of a LOXCH4 Rocket Engine Demonstrator", IAC-14,C4,1.4×25001, 65th International A stronautical Congress, Toronto, Canada, (2014).

3. P. Natale, F. B attista, "FSB B Test Campaign. Test D ata Assessment Report", HNEW -0088CIRA-DTS-17-0874, issue1, 30th N ovember (2017). 